\title{
VIRTUAL RESTORATION OF THE GREAT WALL BASED ON THE EVIDENCES SCALES, TAKE THE NINE-EYES WATCHTOWER AS AN EXAMPLE
}

\author{
Zongfei $\mathrm{Li}^{1,3}$, Miaole $\mathrm{Hou}^{2,3} *$, Youqiang Dong ${ }^{2,3 *}$, Jian Wang ${ }^{2,3}$, Yuhang Ji ${ }^{2,3}$, Pengpeng Huo ${ }^{2,3}$
}

1 Architecture Department, Beijing University of Civil Engineering and Architecture, No.1, Zhanlan Road, Xicheng District, Beijing 100044, China; lzfbucea@163.com

2 School of Geomatics and Urban Spatial Informatics, Beijing University of Civil Engineering and Architecture, Beijing 100044, China; wangjian@bucea.edu.cn(J.W.); 2108521519033@stu.bucea.edu.cn (Y.J.); 2208521518001@stu.bucea.edu.cn (P.H.)

3 Beijing Key Laboratory For Architectural Heritage Fine Reconstruction \& Health Monitoring, No.15Yongyuan Road, Daxing District, Beijing, 102616

* Correspondence: houmiaole@bucea.edu.cn, dongyouqiang@bucea.edu.cn

KEY WORDS: The scale of evidence, The virtual restoration framework, Restoration scheme, 3D virtual restoration model.

\begin{abstract}
:
Aiming at solving the problem of how to obtain the different evidence and deduce the disappeared sections of the Great Wall site, this paper quoted the concept of evidence scale and proposed a virtual restoration framework. In this paper, taking the Nine-eyes watchtower as an example, we modified the evidence-scale from 10 to 3: the existed relic, the direct evidence and the indirect evidence. The framework consisted of three parts, namely, the classification of evidence scale, the evidence checks and the generation of three-dimensional(3D) virtual models. Base on the support of the restoration scheme and 3DMAX technology, the disappeared parts of Nine-eyes watchtower was finally restored to its original appearance. The result of virtual restoration could not only help the tourists to visit the original appearance of cultural heritage, but also experience the culture value and historical vicissitude. Therefore, it was of great scientific significance and cultural value to study how to carry out virtual restoration for the ancient relics.
\end{abstract}

\section{INTRODUCTION}

The Great Wall with a history of about 2,700 years was one of the most impressive cultural heritages and was well known as one of the greatest wonders in the world. In 1987, it was inscribed as the United Nations Educational, Scientific and Cultural Organization (UNESCO) World Heritage Sites. Suffering from the natural disasters and man-made destruction, many sections belong to the Great Wall have been damaged or disappeared(Li 2008). The protection for the Great Wall has been in imminent(Liu et al. 2020; Wei 2012).

The Nine-eyes watchtower of Jiankou Great Wall was built in the early Ming Dynasty and located in Sihai Town, Yanqing
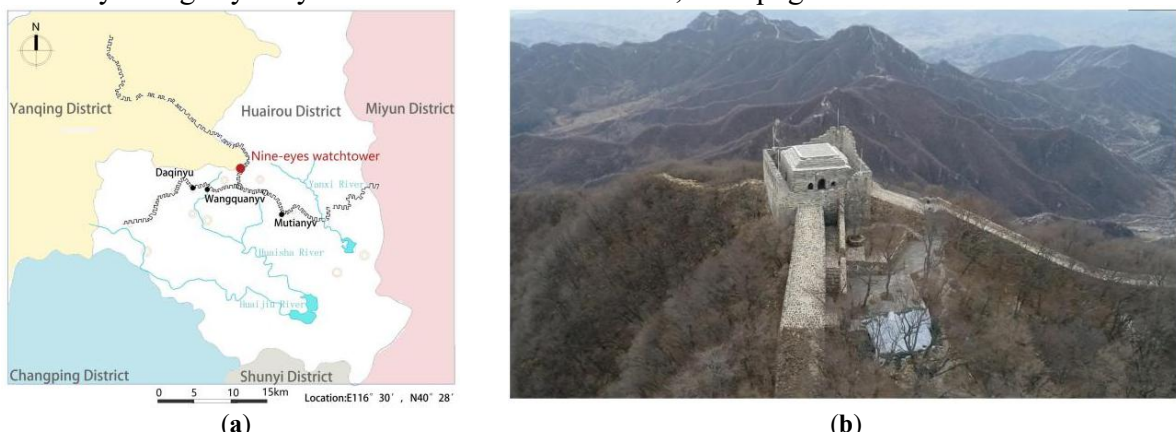

(b)

District, Beijing (Figure 1). In history, the original form of Nine-eyes watchtower had three sections: the base, the first floor and the second floor, but now there were only the base and first floor left, with a residual height of about $10.6 \mathrm{~m}$ and a width of about $13.1 \mathrm{~m}$ (Yanqing County Gazetteer Editorial Committee. 1993). In 2014, a salvage protection project was carried out to prevent this defence tower from collapsing following the principle of "repaired the old as old". By now, the existed Nine-eyes watchtower was shown in Figure 1b. Figure 1c showed the current collapsed state of Nine-eyes watchtower. The disappeared parts included the structure of floor on the second floor and the upper sentry house.

Figure 1. (a)Location of the Nine-eyes watchtower; (b) The status of the Nine-eyes watchtower; (c) The collapsed parts of the Nineeyes watchtower.

Nowadays, the protection methods for the Great Wall could be divided into two categories: the preventive conservation and the salvage conservation according to the Comprehensive Conservation Plan to the protection of Great Wall published by National Cultural Heritage Administration (NCHA). A plenty of the works have been publised about these two protection methods( $\mathrm{Yu}$ 2018; Liu et al. 2016). With the development of the survey technology and computer science

\footnotetext{
* Corresponding author: houmiaole@bucea.edu.cn (Miaole Hou), dongyouqiang@bucea.edu.cn(Youqiang Dong)
} 
and the promotion of the data processing(Pesci et al. 2012; Pritchard et al. 2017; Li, Zhao 2018), the digital technology played an important role in the protection of the the Great Wall and several researchers have developed a consensus on using this type of methods to promote the protection work for the Great Wall.

The current digital protection methods for the Great Wall mainly focused on how to preserve the 3D information and the textures of the Great Wall (Li, Cao 2008) or made use of the digital technology to solve the current problems such as the detection of the diseases(Eldon and Hylton 2005; Barazzetti 2016 ). However, the medium and long-term target task of the Comprehensive Conservation Plan to the protection of Great Wall hoped to build 5 to 10 Great Wall Tourism Denstration Area which have good protection of cultural relics, good environment governance, good display and good service level in the future 10 years, so that the Chinese Great Wall culture would be popular in the world. Hence, the digital restoration would constitute a long tern support for the spread of Great Wall culture.

With the development of the survey technology and computer science, the digital restoration would constitute a long tern support for the spread of Great Wall culture. The virtual restoration could recover the damaged culture relics to the former state at a specified time node in computer(Li 2006). The result of virtual restoration could not only help the tourists to visit the original appearance of cultural heritage, but also experience the culture value and historical vicissitude. Therefore, it was of great scientific significance and cultural value to study how to carry out virtual restoration for the ancient relics. But there were two key limitations should be overcome in this process. One hand, how to obtain and organize evidence was a primary problem(Denker 2019). On the other hand, how to deduce the disappeared sections of the Great Wall should be another key issue to consider. This was because the virtual restoration involved different aspects of the evidence type.

\section{METHODOLOGY}

\subsection{Proposed solution}

To solve the problem of single evidence source, we reversed this evidence scale which was initially applied to the evaluation of the restoration results of the Byzantium 1200 to collect the evidence of the Nine-eye watchtower here(Ortiz-Cordero et al. 2018). We modified the evidence-scale from 10 to 3 : the existed relic, the direct evidence and the indirect evidence(Figure 2). The existed relics can provide the most reliable evidence for the virtual restoration, such as the form, the structure, the materials, and the craftsmanship information. The evidence which directly described the Nine-eyes watchtower was defined as the direct evidence. The indirect evidence were the supplements to the existed relics and the direct evidence. We classified the indirect evidence into two categories: the evidence from the official literatures and the evidence from other defence tower.

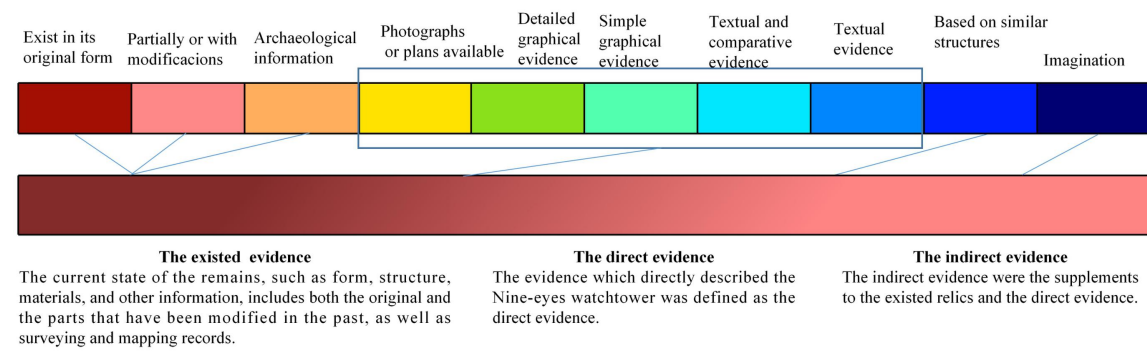

Figure 2. The classification of the evidence scale.

To ensure the scientificity and authority of the evidence, these evidences were separately checked to verify validity. Base on the above, the idea of multi-evidence fusion was used to deduce the disappeared parts of Nine-eyes watchtower, then restoration scheme was restored to show it, $\mathrm{s}$ original appearance. Finally, with the support of the restoration scheme and 3DMAX technology, the virtual restoration study was realized. This research had a great advantage. The result of virtual restoration could not only help the tourists to visit the original appearance of cultural heritage, but also experience the culture value and historical vicissitude. Therefore, it was of great scientific significance and cultural value to study how to carry out virtual restoration for the ancient relics.

As was shown in Figure 3, our proposed a framework of the virtual restoration for the Nine-eyes watchtower that consisted of three parts:(1) the collection of restoration evidence based on a modified scale of evidence; (2)the virtual restoration scheme deduction by the process of evidence check for the architectural form, structure, material and craftsmanship; (3)the 3D model generation of the Nine-eyes watchtower.

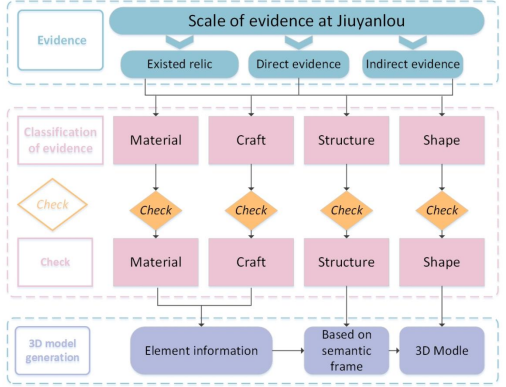

Figure 3. The framework of the virtual restoration for the Nine-eyes watchtower.

\subsection{The whole scheme of deducing results}

One of the goals was to recover the floor structure of the Nineeyes watchtower. We have confirmed that the second floor had a brick-wood structure to support the upper sentry house. Here, the Chinese transitional brick-wood structure principle was used to deduce the floor structure. As shown in Figure 4a, the wooden pillars were inserted into the reserved holes of wall, wooden beams were placed on the top of the pillars, then planks were tidily paved on the beams. By the structural organization, the brick-wood structure was steadily placed on the top of corridor with the shape of " 回 ". The spouts were 
arranged at the corners of the middle platform as was shown in Figure $4 \mathrm{~b}$. The rainwater from the second-floor flowed into the drainage ditch through spouts and drained into the corridor.

Another goal of virtual restoration was to restore the architectural form and interior space type of the sentry house.The Figure 5a depicted the form of sentry house that was a Flush Gable Roof with gray round tile. The Wang shou and Pao shou were separately placed on the main ridge and vertical ridge of the Flush Gable Roof. As shown in figure 5b, the interior space of the sentry house was a typical tube arch structural space. The type of tube arch was consistent with the existed tube arch structure of the first floor and inherited the universal craftsmanship for creating the tube arch space in Ming Dynasty. The advantage of tube arch structural space was not only made use of bricks arch to achieve the balance of the roof weight but also can create a spacious interior space.

Finally, through evidence examination and reasoning, a virtual restoration scheme of Nine-eyes watchtower was proposed based on the idea of multi-evidence fusion. As was shown in Figure 6, this scheme included several of sections: plane drawing, facade drawing, cross-section drawing and the appearance of Nine-eyes watchtower.

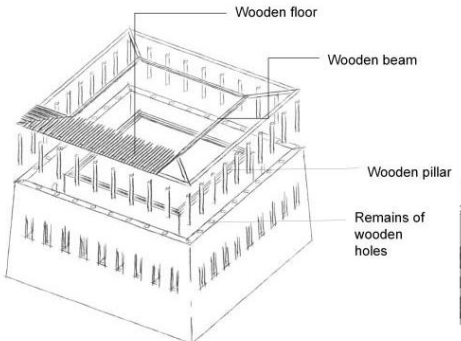

(a)

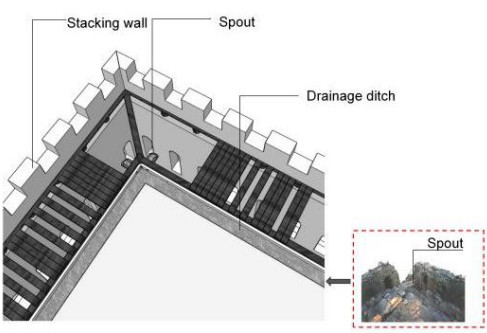

(b)

Figure 4. Restoration diagram of floor structure: (a) The restoration diagram of the brick-wood structure; (b) The brick-wood structure was placed on the top of corridor with the shape of "回".
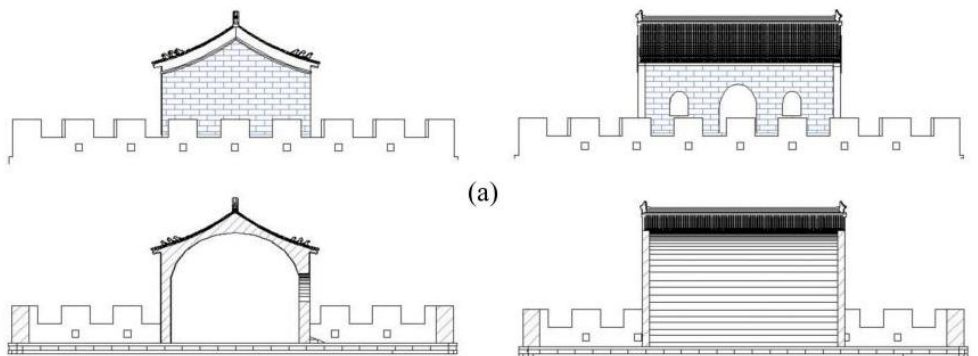

(a)

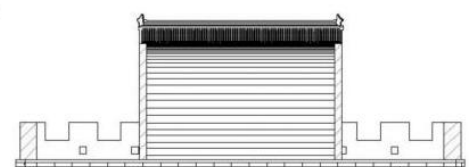

(b)

Figure 5. The architectural form and interior space type of the sentry house: (a) The facade drawing of sentry house with Flush Gable Roof and gray round tile; (b) The cross-section drawing of the tube arch space of sentry house.

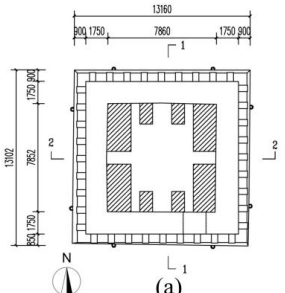

(a)

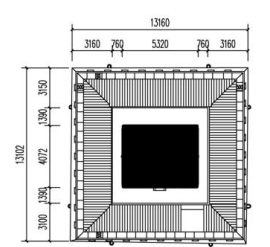

(b)

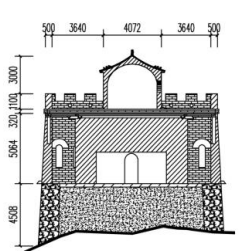

(c)

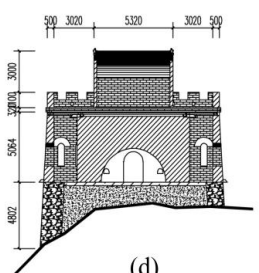

(d)

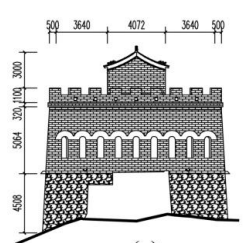

(e)

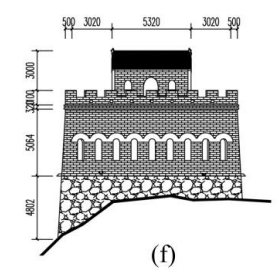

(f)

Figure 6. The virtual restoration scheme of Nine-eyes watchtower: (a) The plane drawing of the first floor; (b) The plane drawing of the second floor; (c) The cross-section drawing ,1-1; (d)The cross-section drawing ,2-2; (e)The west facade;(f) The south facade.

\section{THE VIRTUAL RESTORATION PROCESS AND RESULTS}

Based on the above inferences, the deduction results had been obtained for the floor structure, the form of the sentry house, internal space, and restoration scheme which had been designed for the virtual restoration. In the following sections, the drawings which could reflect the characteristics of Nine-eyes watchtower would be shown for the virtual restoration.
(1)The craftsmanship of virtual restoration should keep the same with the realistic restoration for the Nine-eyes watchtower. As shown in Figure 7a, the craftsmanship of wall was one-shun-one-ding and the arrow window was arch. As shown in Figure 7a,b, the lost arrow windows and wall of Nine-eyes watchtower were gradually recover by using the arch and the craftsmanship of one-shun-one-ding along the traces of collapsed arrow windows. During the virtual restoration process, the size of new arrow window and wall were in accordance with the original geometric information of the Nine-eyes watchtower. 


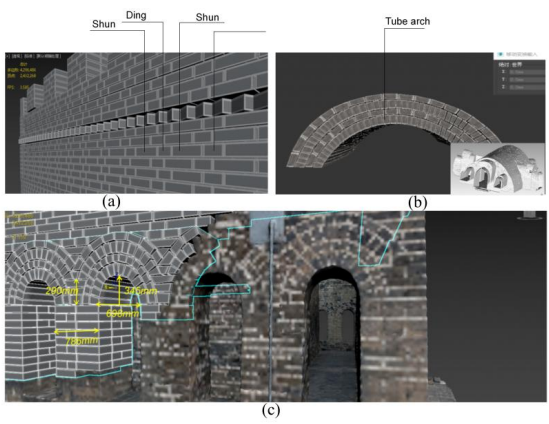

Figure 7. (a) The craftsmanship of wall; (b) The craftsmanship of arrow window; (c) The geometry information of arrow windows.

(2) The next task was to restore the floor struction and the accessorial structures such as brick eaves, observation ports. The size of restoration was strictly keep to the Nine-eyes watchtower relic as seen in Figure 8. In 3DMAX, the pillar, beam and plank were combined into the brick-wood structure which was supported the sentry house on the second floor. On the second floor, the ladder well was reserved for soldiers to pass through. In addition, the accessorial structures such as brick eaves, observation ports were restored in sequence(Figure 9).

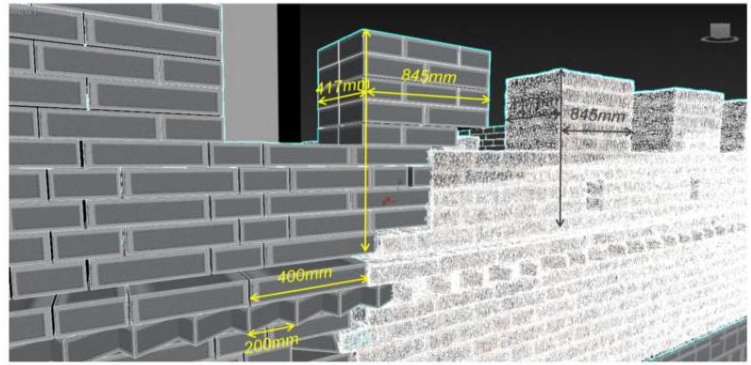

Figure 8. The geometry information of battlement restoration.

(3)Finally, according to the restoration scheme, we separately edited flat-tile, round-tile, and decorative components in 3DMAX, such as Wang shou, Pao shou. Then those plugins gradually insert on the roof by a virtual process (Figure 10). The result of virtual restoration included not only the model of the Nine-eyes watchtower, but also its virtual scene. The researchers could view the Nine-eyes watchtower from different perspective, which contained the Nine-eyes watchtower and the environmental elements such as mountain and trees(Figure 11 ).

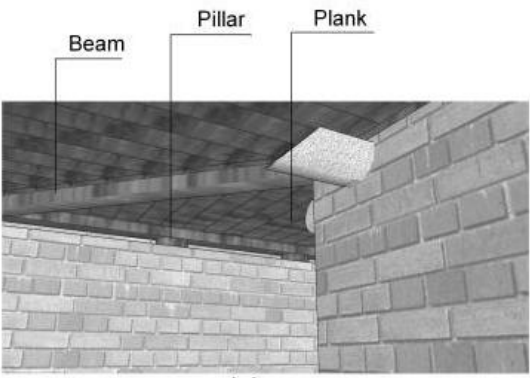

(a)

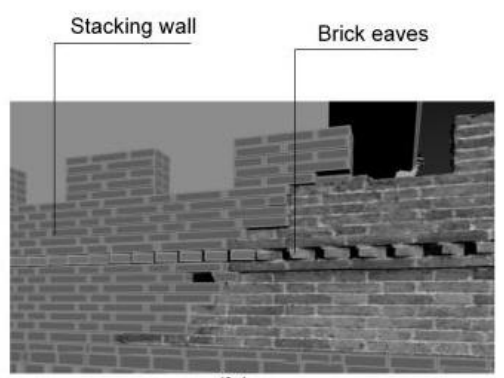

(b)

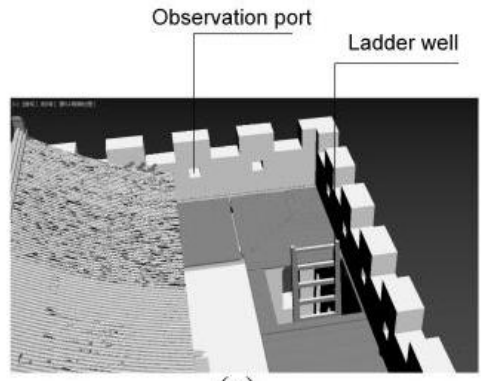

(c)

Figure 9.The details of virtual restoration:(a) The floor structure of the brick-wood structure type; (b) The accessorial structures of battlemen; (c) The details of the second floor.

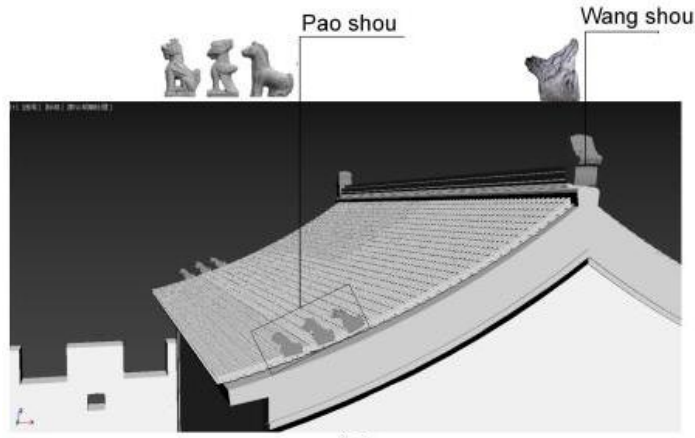

(a)

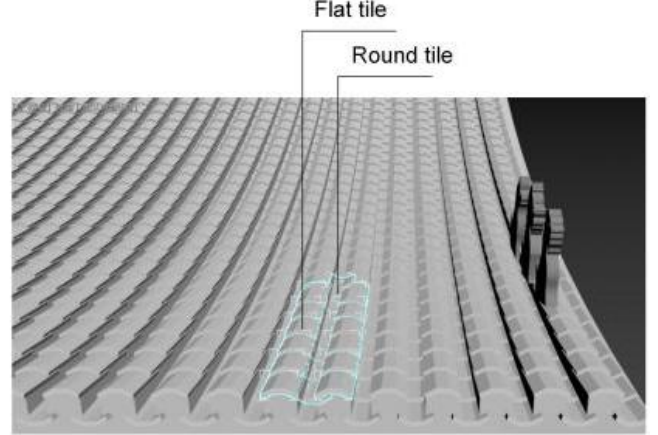

(b)

Figure 10.The virtual restoration of roof and decorative elements. 


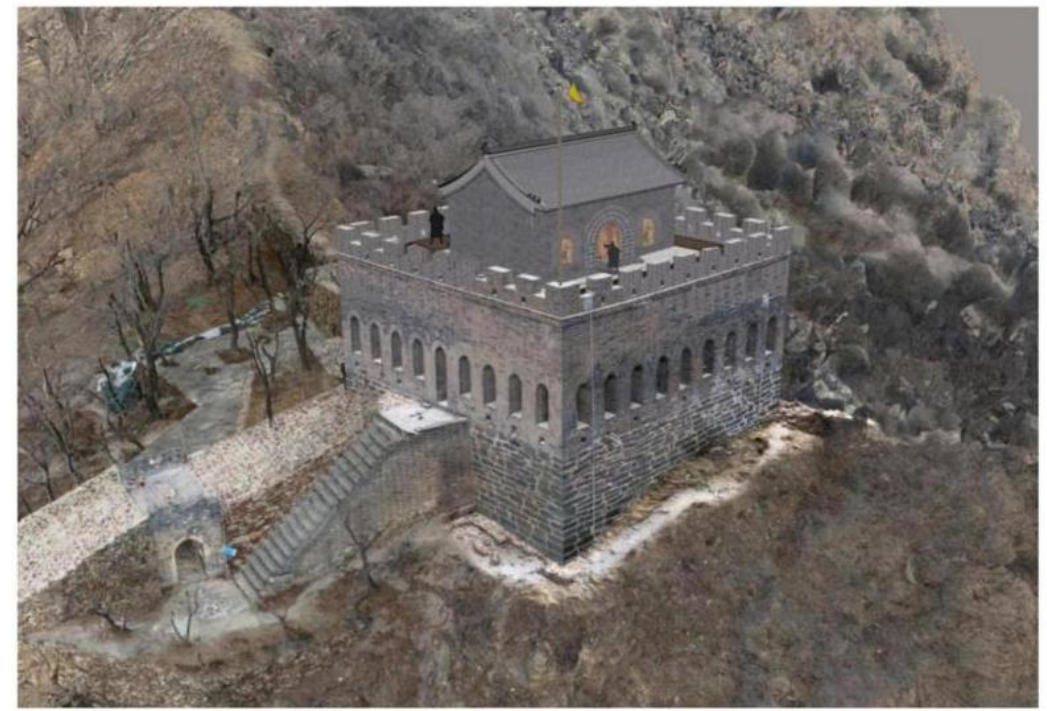

Figure 11.The results of virtual restoration for Nine-eyes watchtower.

\section{CONCLUSION}

This study more clearly confirmed that even if ancient relics of like the Great Wall experienced vicissitudes and collapse, they could still be achieved the virtual restoration through the framework of virtual restoration.

It was confirmed that modern surveying and mapping methods played an important role to support virtual restoration for ancient relics, and the accuracy of the virtual restoration was depended on the data acquisition technology. For example, the fine geometrical information of Nine-eyes watchtower could be efficiently collected based on UAV photogrammetry technology, 3D laser scanning technology. In the field of ancient relics protection, the idea of interdisciplinary integration have renewed the new vitality for the virtual restoration of cultural heritage. With the joint supporting of Architecture, Surveying and Mapping and Computer, the feasibility of virtual restoration research have been successfully verified.

\section{ACKNOWLEDGEMENTS}

This work is supported by Youth Beijing Scholar Program, Scientific Research Program Project of Beijing Education Commission (KM202110016005) and Natural Science Foundation of Beijing Province (KZ202110016021).

\section{REFERENCES}

Denker, A. 2019. Resonation of the vitruvius's modular, systematic approach with the computational mindset of the digital age: $3 \mathrm{~d}$ modeling of the ionian temples of aegean turkey. doi:10.5194/isprs-archives-XLII-2-W15-389-2019.

Li, C.L., 2006. Virtual restoration of axisymmetric relic fragments. JOURNAL OF COMPUTER AIDED DESIGN AND COMPUTER GRAPHICS, 18(5), 620.

Li, X.J, et al. 2008. Research on the damage of the Great Wall of Ming Dynasty in Beijing by remote sensing. Science in China Series E: Technological Sciences 51.1: 195-202.
Li, Y.W., H. R. Zhao. 2018. Comprehensive Display Design of the Great Wall' s Cultural Landscape Based on Virtual Environment. Bulletin of Surveying and Mapping (08),131$135+149$.

Liu, et al. 2016. Discussion on large sites conservation planning taking Great Wall of Ming Dynasty Qinghai Datong section as an example. Shanxi Architecture 42.6: 3-5.

Liu, W.Y., et al. 2020. Great Wall of China Conservation Report (2017-2018). Chinese Cultural Heritage. (02):91-102.

Ortiz-Cordero, R., Pastor, E. L., Fernández, R. E. H. 2018. Proposal for the improvement and modification in the scale of evidence for virtual reconstruction of the cultural heritage: A first approach in the mosque-cathedral and the fluvial landscape of Cordoba. Journal of Cultural Heritage, 30, 10-15.

Pesci, A. , Bonali, E. , Galli, C. , \& Boschi, E. 2012. Laser scanning and digital imaging for the investigation of an ancient building: palazzo d'accursio study case (bologna, italy). Journal of Cultural Heritage, 13(2), 215-220.

Wei, Y. 2012. Investigation of the Ming Dynasty Great Wall Resources in Ningxia Based on Digital Photogrammetry. Geomatics \& Spatial Information Technology. (10),199$200+204+207$.

Yu, B. 2018. The overall protection and management of the Great Wall of China: challenges and explorations . Chinese Cultural Heritage. (03): 31-40. 